\title{
Vortex Reconnections and Rebounds in Trapped Atomic Bose-Einstein Condensates
}

\author{
Simone Serafini, ${ }^{1}$ Luca Galantucci, ${ }^{2}$ Elena Iseni, ${ }^{1}$ Tom Bienaimé, ${ }^{1}$ Russell N. Bisset, ${ }^{1}$ Carlo F. Barenghi, ${ }^{2}$ \\ Franco Dalfovo, ${ }^{1}$ Giacomo Lamporesi, ${ }^{1, *}$ and Gabriele Ferrari ${ }^{1}$ \\ ${ }^{1}$ INO-CNR BEC Center and Dipartimento di Fisica, Università di Trento, 38123 Povo, Italy \\ ${ }^{2}$ Joint Quantum Centre (JQC) Durham-Newcastle, and School of Mathematics and Statistics, \\ Newcastle University, Newcastle upon Tyne NE1 7RU, United Kingdom \\ (Received 27 January 2017; revised manuscript received 10 April 2017; published 25 May 2017)
}

\begin{abstract}
Reconnections and interactions of filamentary coherent structures play a fundamental role in the dynamics of fluids, redistributing energy and helicity among the length scales and inducing fine-scale turbulent mixing. Unlike ordinary fluids, where vorticity is a continuous field, in quantum fluids vorticity is concentrated into discrete (quantized) vortex lines turning vortex reconnections into isolated events, making it conceptually easier to study. Here, we report experimental and numerical observations of threedimensional quantum vortex interactions in a cigar-shaped atomic Bose-Einstein condensate. In addition to standard reconnections, already numerically and experimentally observed in homogeneous systems away from boundaries, we show that double reconnections, rebounds, and ejections can also occur as a consequence of the nonhomogeneous, confined nature of the system.
\end{abstract}

DOI: 10.1103/PhysRevX.7.021031

Subject Areas: Atomic and Molecular Physics

\section{INTRODUCTION}

The interaction and reconnection of filaments are key aspects in the description of the dynamics of fluids [1-3], plasmas [4-6], nematic liquid crystals [7], macromolecules [8] (including DNA [9]), and optical beams [10,11]. In quantum fluids, vortices are topological defects of the system's order parameter, around which the circulation of the velocity field is quantized [12-15]. Their discrete filamentary nature makes quantum fluids an ideal setting for the study of vortex interactions and reconnections. In particular, reconnections trigger a turbulent energy cascade [16] in which vortex lines self-organize in bundles [17], creating the same Kolmogorov distribution of kinetic energy over the length scales, a signature of a cascade mechanism that is observed in ordinary turbulence [16,18-20]. Cascade processes are central in turbulent motions. A related cascade of wavelike excitations was in fact recently observed in the momentum distribution [21], with an exponent consistent with predictions of waveturbulence theory [22]. Reconnection events also impact the evolution of the flow's topology [23], redistributing helicity among length scales [24,25]. Finally, in the lowtemperature limit, reconnections are the ultimate process of dissipation of superfluid kinetic energy since they trigger a Kelvin wave cascade [26,27] that turns incompressible

\footnotetext{
*giacomo.lamporesi@ino.it
}

Published by the American Physical Society under the terms of the Creative Commons Attribution 4.0 International license. Further distribution of this work must maintain attribution to the author(s) and the published article's title, journal citation, and DOI. kinetic energy into acoustic modes [28], hence, heating. Previous experimental [29,30], theoretical [31], and numerical [32-38] studies of reconnections have been performed in homogeneous systems away from boundaries.

Here, we focus on elongated Bose-Einstein condensates (BECs) of ultracold atoms confined by magnetic harmonic potentials, ideal systems that allow for different regimes of three-dimensional vortex-vortex interactions in the close presence of boundaries. Anisotropic boundaries induce vortical filaments to preferentially align along the shortest direction, minimizing energy. In flat, cylindrically symmetric, disk-shaped condensates, vortices are the shortest when aligned along the axis of symmetry, moving along two-dimensional trajectories clockwise or anticlockwise, depending on their sign [39-44]. Instead, vortices in cylindrically symmetric, cigar-shaped condensates are the shortest when they lie on radial planes. Moreover, the boundaries affect the structure of the vortical flow [45-48] in such a way that two vortices interact only when their minimum distance is within a range of the order of the transverse size of the condensate.

In the present work, an innovative imaging technique, exploiting self-interference effects of outcoupled atoms, is introduced in order to extract both the position and orientation of $3 \mathrm{D}$ vortex lines from a temporal sequence of absorption images. We then combine experiments and numerical Gross-Pitaevskii (GP) simulations to study the interaction between two vortex lines approaching at various relative speeds and angles. Our experiments and simulations show that the interaction between vortex lines in a finite system is rather different from the one in infinite uniform superfluids. Boundary-induced effects, such as rebounds, double reconnections, and ejections, are discussed here in 
detail. These types of processes may play an important role in the dynamics of trapped condensates in multivortex and turbulentlike configurations, and, on a wider perspective, they can represent novel keys for better understanding the behavior of superfluids near boundaries.

\section{EXPERIMENT}

\section{A. Preparation of BECs with vortices}

Experimentally, we confine sodium atoms in an elongated cigar-shaped harmonic magnetic trap with axial and radial frequencies $\omega_{x} / 2 \pi=9.2 \mathrm{~Hz}$ and $\omega_{\perp} / 2 \pi=92 \mathrm{~Hz}$, respectively. By means of a radio-frequency forced evaporation, the cold atomic sample undergoes the BEC transition and, in the end, condensates containing about $N_{0}=2 \times 10^{7}$ atoms and a negligible thermal fraction $\left(T<150 \mathrm{nK}, T_{c} \simeq 500 \mathrm{nK}\right)$ are obtained. Thanks to the Kibble-Zurek mechanism [49,50], the temperature quench through the BEC transition [40,41,51,52] produces different phase domains in the order parameter of the system that quickly evolve into topological defects. In our geometry, these defects are vortex lines mainly oriented in the transverse direction, as those predicted in Refs. [45,46] and characterized in Ref. [47]. Similar vortices can be obtained as decay products of phase imprinted dark solitons in a BEC [53] or a Fermi superfluid gas [54,55]. Here, we use a cooling rate of $4 \mu \mathrm{K} / \mathrm{s}$ in order to produce, on average, two vortices in each condensate at the time when the observation starts, about $250 \mathrm{~ms}$ after the phase transition. Such vortices move in the nonrotating condensate and can be directly imaged in real time [41,56,57]. In comparison, individual vortex visualization in superfluid helium is more intrusive, requiring tracer particles whose diameter is about $10^{4}$ times larger than the vortex core [58].

\section{B. Sample extraction and real-time imaging}

A new imaging method allows us to follow the vortex dynamics in real time, as sketched in Fig. 1. Similar to Refs. [41,56], a small sample of the atomic system $\left(\sim 10^{5}\right.$ atoms) is repeatedly extracted from the BEC every $12 \mathrm{~ms}$ (up to 75 times). The outcoupled atoms freely expand and fall under the effect of gravity. Each partial extraction is implemented by coupling the trapped state $\left|F=1, m_{F}=-1\right\rangle$ to the nonmagnetic one $|1,0\rangle$ with a radio-frequency field. The energy difference between the two states is spatially dependent because of the inhomogeneity of the trapping potential (see Appendix A).

The novelty of our technique is represented by the fact that the rf field is frequency swept linearly in time in order to match the resonant condition at different positions throughout the BEC, from top to bottom. An important point to note is that the phase of the released atoms evolves more slowly because they do not feel the trapping potential. As a consequence, the wave function of the outcoupled atoms experiences constructive or destructive self-interference effects, depending on the phase difference accumulated between the early-released (upper) and late-released (lower) atoms and how this relates to the in situ phase on different sides of the vortex core. We use the GP equation to simulate the radio-frequency extraction in order to determine how a vortex with given position and orientation in the trapped $\mathrm{BEC}$

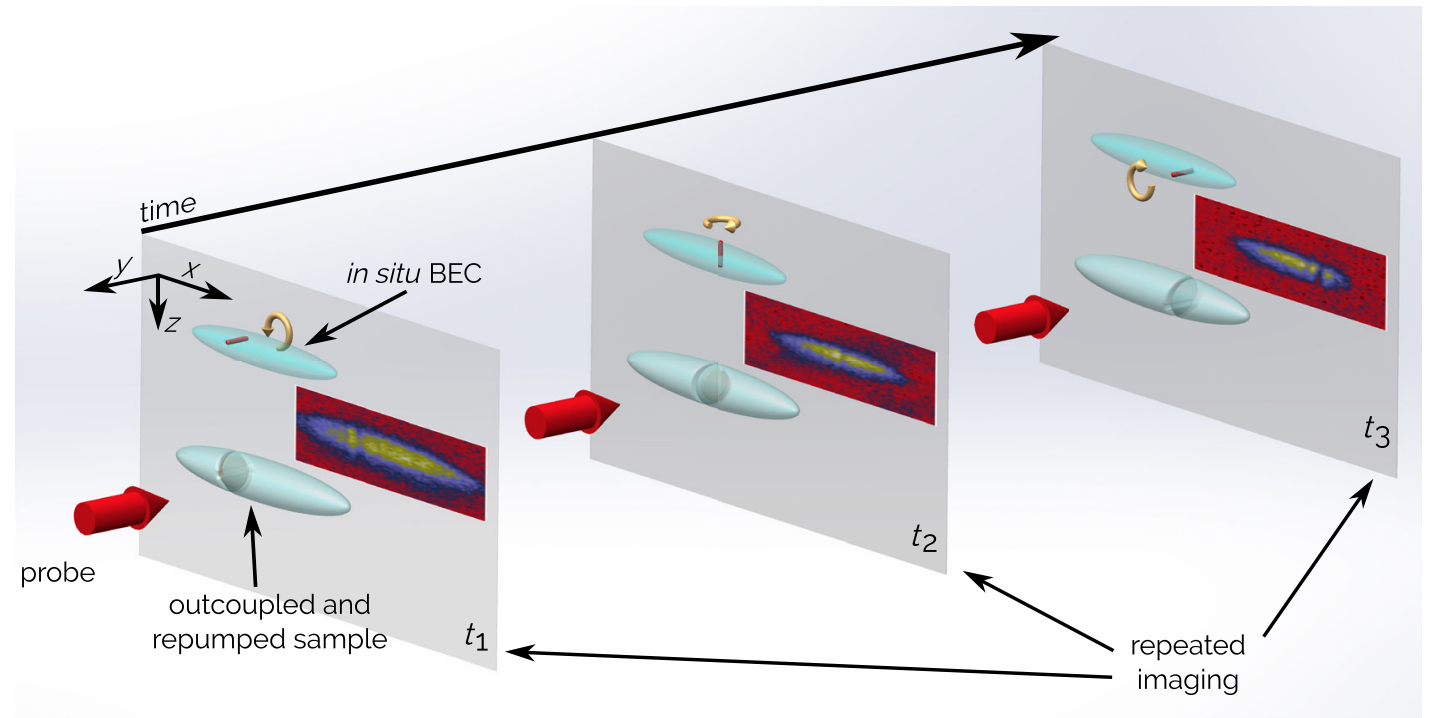

FIG. 1. Sketch of an imaging sequence. A trapped condensate (smaller, light blue ellipsoid) contains a transverse vortex line that moves and rotates around the trap center; the direction of the atomic flow around the vortex filament is indicated by the yellow arrow. A small fraction of atoms is repeatedly extracted, typically every $12 \mathrm{~ms}$; these atoms expand and fall in the gravity field, and are imaged in absorption by a probe laser beam after they are spatially separated from the trapped condensate. Each absorption image contains the essential features associated with the vortex lines. 
manifests itself in the observed density distribution of the outcoupled atoms after expansion (see Appendix C).

A microwave field remains on to transfer the extracted atoms from $|1,0\rangle$ to $|2,0\rangle$, which is detectable with the probe light. The resonant condition for the transfer is matched at $z_{r} \approx 280 \mu \mathrm{m}$ below the trapped BEC, far enough to leave it unaffected. We probe the extracted atoms via standard absorption imaging after $13 \mathrm{~ms}$ of total time of flight at $z_{i} \approx 830 \mu \mathrm{m}$ below the trap center. Such a time of flight is enough for vortices to become visible with our imaging resolution.

\section{Data analysis}

Each absorption image [Fig. 2(a)] is integrated radially along the $z$ axis and the axial profile is obtained. By fitting the latter, we calculate the density residuals [see Fig. 2(b)]. This procedure is performed on each extraction and then

(a)
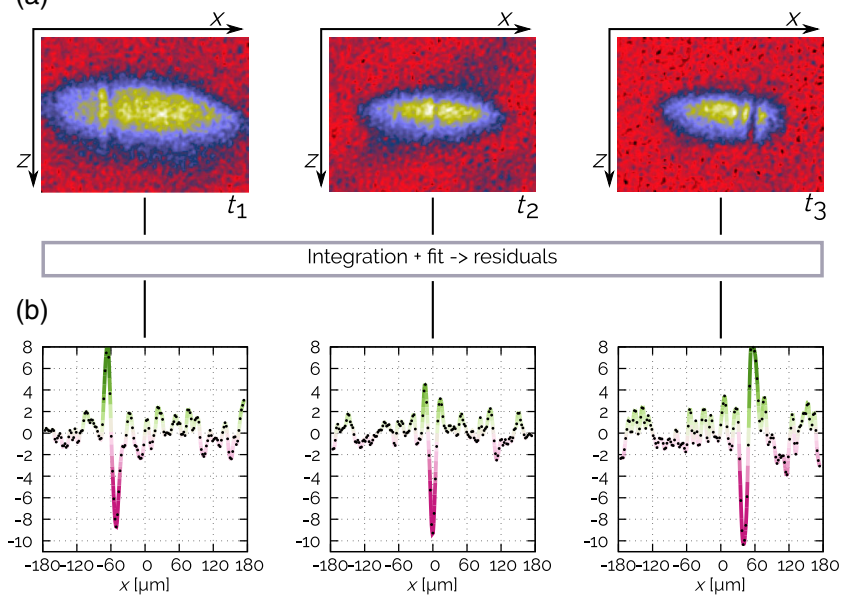

(c)

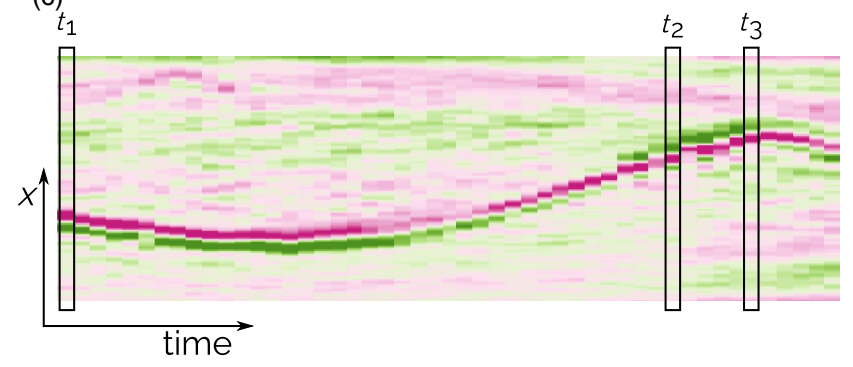

FIG. 2. (a) Examples of absorption images of the outcoupled atoms (the same as in Fig. 1). The vortex axial position is clearly visible. (b) After integrating radially and fitting the absorption images, we determine the residuals, which exhibit minima (pink) and maxima (green) due to interference effects among atoms that are outcoupled from the trapped condensate at different places and times. (c) Full temporal sequence of residuals for a given condensate, showing the real-time evolution of a vortex which moves axially and rotates around the $x$ axis, from an initial orientation along $y$ (green-pink) at $t_{1}$ to an orientation along $z$ (green-pink-green) in $t_{2}$ and then along $-y$ (pink-green) at $t_{3}$. The relation between the shape of the residuals and the orientation of the vortex is extracted from numerical simulations. the full temporal sequence is reconstructed in order to follow the vortex trajectories in the trapped condensate, as in Fig. 2(c).

Thanks to the abovementioned self-interference effect, if a vortex is present, the density residuals show a strong local deviation from the unperturbed distribution, as in Fig. 2(b), and the fit allows us to extract information on the vortex axial position, as well as on its orientation in the radial plane at any given time (see Appendix $\mathrm{C}$ for details).
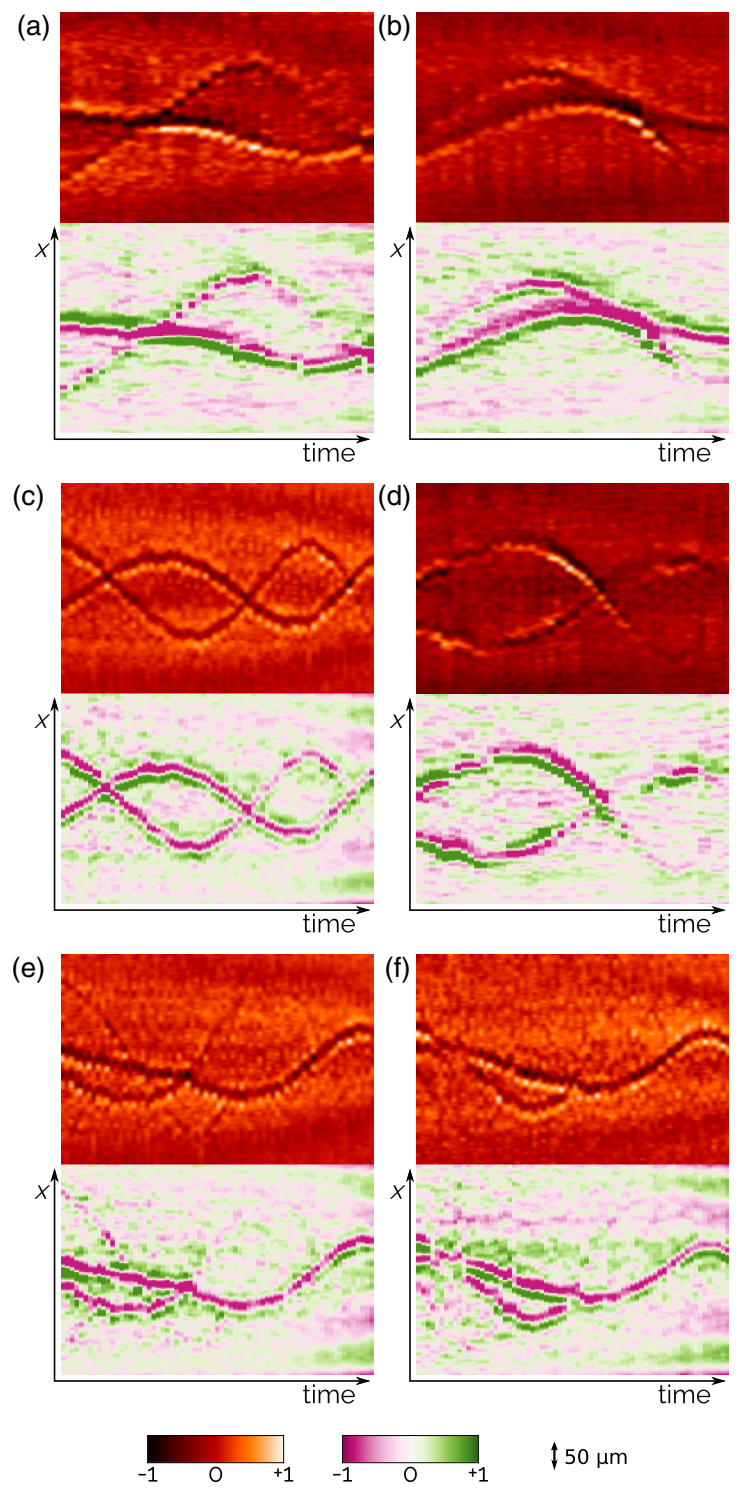

FIG. 3. Examples of different interaction mechanisms observed in the case of two approaching vortices. Each temporal sequence is shown twice with two different color palettes; the red palette enhances the contrast, so that also vortices close to the edges can be seen, whereas the pink-green palette better illustrates the vortex orientation in the radial plane. (a),(b) Vortices approach and bounce back, (c) their axial trajectories intersect preserving visibility and orientation, (d) they cross, producing sudden changes of visibility, and (e),(f) the visibility of one vortex is almost completely lost after interacting with the other. 
Figure 3 shows examples of the temporal evolution of the density-residual profiles in BECs containing two vortices. Two different color palettes are used in order to extract different pieces of information. The red palette best highlights the trajectory contrast. One can track the vortex axial location in time and, hence, determine the orbit amplitude and the axial velocity. Notice that in some cases, very faint trajectories (corresponding to vortices close to the BEC surface) can also be seen. It is also possible to understand how the vortex line is oriented in the radial plane and how it rotates about the long axis of the condensate. The diverging pink-green palette helps to visualize the shape of the density modulation from which one can better track the vortex orientation in time. From numerical simulations we infer that, at least when the orbiting parameter is not too large, the vorticity points along $y$ if the interference pattern is greenpink along $x$ [see Fig. 2(b)]; its antivortex configuration, oriented toward $-y$, corresponds to a pink-green pattern; the symmetric pattern green-pink-green is obtained when the vortex is aligned perpendicularly to the imaging direction, a vortex oriented along $+z$ providing the same density residual as one oriented along $-z$.

\section{NUMERICAL SIMULATIONS}

In order to gain closer insight into vortex interactions, we perform numerical simulations by using the GrossPitaevskii equation $[59,60]$ for a BEC at $T=0$. Temperature effects are expected to be small. In a previous work [57], we have already observed that the dynamics of single vortices is very weakly affected by thermal excitations. This is expected to be true also for vortex-vortex interaction processes occurring in the central region of our BEC, where the thermal density is negligible. There is also evidence that thermal excitations do not affect the rapid motion of vortex lines during the reconnections [61].

We track the vortices by employing an algorithm based on the pseudovorticity vector, achieving subgrid resolution (see Appendix B). Since the experimental BECs are too large for our computational resources, we simulate smaller BECs $\left(\sim 4 \times 10^{5}\right.$ atoms); this implies a reduction of the ratio $R_{\perp} / \xi$ by a factor of 3 , where $\xi$ is the healing length and $R_{\perp}$ is the transverse Thomas-Fermi radius. However, such a difference does not affect the qualitative comparison between experiment and simulations.

If we imprint a single straight vortex line off center on a radial plane, we find that it orbits around the center of the condensate $[39,41]$ along an elliptical orbit that is orthogonal to the vortex line itself. The orbit, which is a trajectory of constant energy [62] and an isoline of the trapping potential, is uniquely determined by the orbit parameter $\chi=r_{0} / R_{\perp}=x_{0} / R_{x}$, where $r_{0}$ and $x_{0}$ correspond to the radial and axial semiaxes of the ellipse, while $R_{x}$ is the axial Thomas-Fermi radius. The orbital period is maximum when the vortex moves on a very small orbit $(\chi \ll 1)$ and corresponds to $T_{0}=8 \pi \mu /\left[3 \hbar \omega_{\perp} \omega_{x} \ln \left(R_{\perp} / \xi\right)\right]$ [57], while it decreases with increasing $\chi$ [62-65].

If instead we imprint two transverse vortices in a given $\mathrm{BEC}$, we find that the evolution can be divided into two stages. In the first stage, when the axial separation of the vortices is larger than $R_{\perp}$, the vortices move almost independently; in the second stage, when the axial separation becomes smaller than $R_{\perp}$, we observe a significant interaction which seems to be determined mainly by the relative orientation $\theta_{\text {rel }}$ and velocity $v_{\text {rel }}$ when they start interacting.

We first perform simulations in which two orthogonal vortices are initially imprinted in radial planes at opposite axial positions $\pm x_{0}$; see Figs. 4(a)-4(c) (orthogonality is chosen because of its maximal dissimilarity with respect to flat 2D systems). Different $x_{0}$ values are chosen, corresponding to different orbit parameters $\chi$ and, hence, to different impact velocities. The early stage can be described as the combination of two single-vortex motions on mutually perpendicular elliptical orbits.

In fact, in an elongated condensate, the superfluid flow of each vortex becomes negligible at distances of the order of $R_{\perp}$ from the line, as can be verified by solving the stationary GP equation. This means that when two vortices are at distances larger than $\sim R_{\perp}$, they behave as noninteracting objects, as is indeed observed in time-dependent GP simulations. This is crucial in order to interpret and classify the vortex-vortex interaction as a collision with well-defined initial and final velocities and orientations. In a different 3D geometry, it would be very difficult to define and control a global "relative velocity and orientation" of a vortex line. If a nonrotating condensate is confined in a spherical potential, or is uniform, for instance, the distance between two vortices and their relative velocity and orientation could be defined only locally: vortices do not possess a preferred orientation, they can be easily bent, and each piece of vortex is affected by a long-range interaction with all other vortices in the condensate. Our geometry instead naturally provides welldefined collision events, such as reconnecting or bouncing lines, occurring in a narrow interaction region.

Only when the minimum distance between the vortices becomes of the order of $R_{\perp}$, the vortices start rotating in the radial plane, attempting to arrange themselves in the preferred (energy-conserving) antiparallel configuration, as shown in Figs. 4(a)-4(c). The axial motion of the vortices towards each other, driven by the inhomogeneous density, is faster if the vortices are close to the condensate's boundary $[62,64,65]$. The antiparallel configuration which the vortices attempt to achieve induces them to drift radially towards the radial center of the condensate. This drift is similar to the well-known self-induced motion of a pair of straight antiparallel vortex lines $[42,43,66]$ in a homogeneous condensate. The balance between the radial and axial motions that we describe determines the features of the second stage of the interaction. 


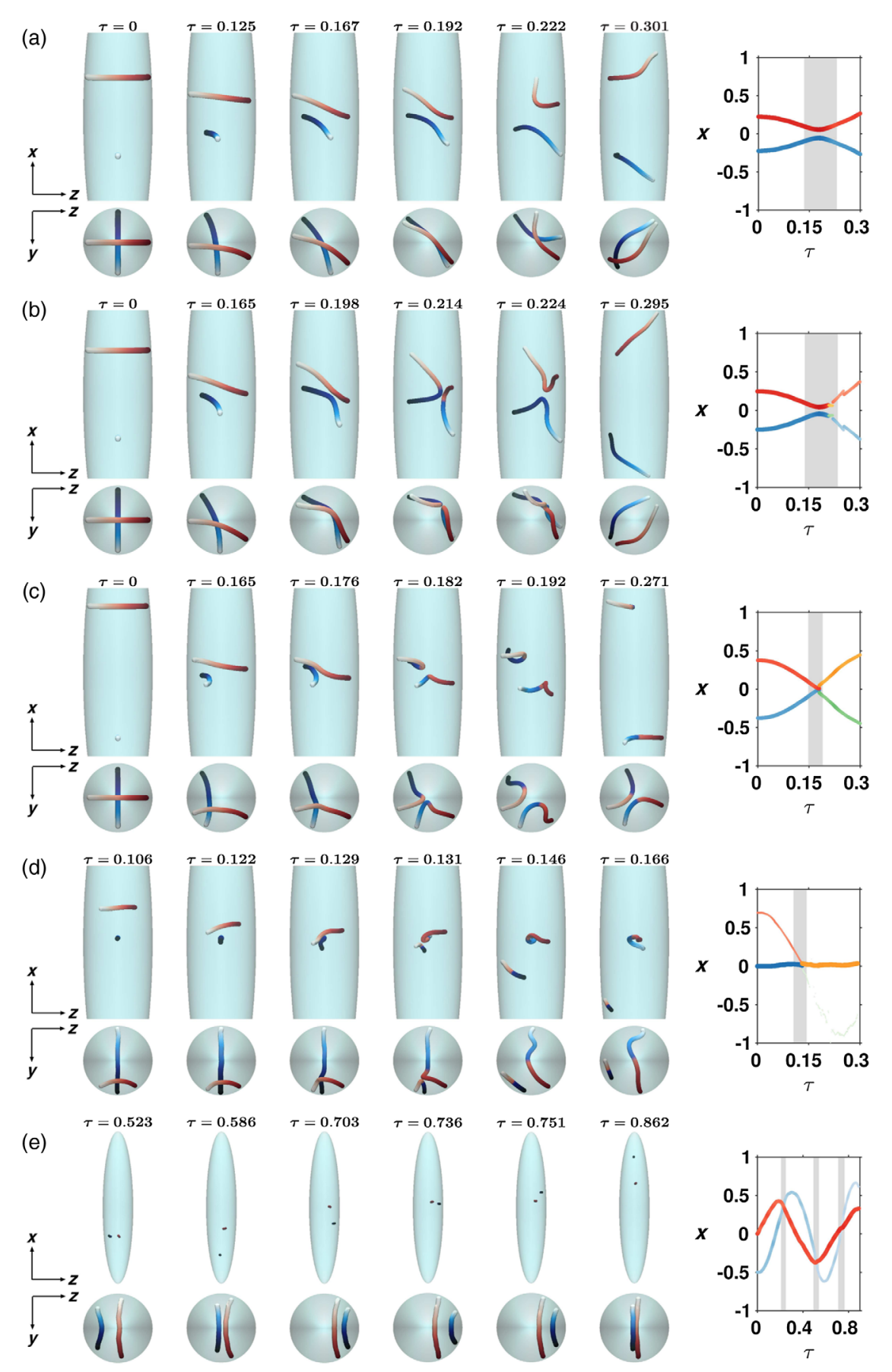

FIG. 4. The first six columns show radial and axial snapshots from the GP simulations of two interacting vortex lines. On the right, the axial coordinate $x$ (in units of $R_{x}$ ) of the center of vorticity of each vortex is plotted versus normalized time $\tau=t / T_{0}$. Initial line colors (red and blue) help identify vortices in the snapshots until they reconnect. After the first reconnection, line colors switch to orange and green and again to red and blue if a second reconnection occurs. Line transparency indicates how visible vortices are expected to be, given their orbit amplitude (see Appendix B for further details on line transparency). The gray region highlights the interaction interval where the minimum distance between the vortices is smaller than $R_{\perp}$. (a)-(c) Perpendicular vortices are imprinted on opposite radial planes with corresponding orbit parameters $\chi=0.22,0.25,0.375$, respectively: (a) illustrates a vortex rebound, (b) shows the double reconnection interaction, with reconnections occurring at $\tau=0.208$ and $\tau=0.221$ (see Appendix B for a zoom on the double reconnection event), and (c) depicts a single reconnection occurring at $\tau=0.179$, with the consequent triggering of Kelvin waves. Panel (d) illustrates a nonsymmetrical reconnection (at $\tau=0.130$ ) between a vortex imprinted on the central plane of the condensate through its center (blue) and a vortex (red) imprinted orthogonally to the first one with a large orbit parameter $\chi=0.7$. One of the reconnected vortices lies on an even wider orbit (larger $\chi$ ), where the BEC density is lower and its visibility becomes consequently greatly reduced. Panel (e) describes the orbiting dynamics between two parallel vortices imprinted on different orbits $(\chi=0.33$, 0.5). Notice that in (a)-(c) the first snapshot corresponds to $\tau=0$, whereas in (d) and (f) the snapshots are all later in time. 
Briefly, if the axial collision velocity is sufficiently high (i.e., if the vortex lines start interacting in a region sufficiently close to the boundary $[62,64,65])$, the two vortices tend to reconnect before reaching the center of the condensate, as in Fig. 4(c).

Vice versa, if the interaction begins in a region sufficiently close to the $x$ axis, the radial motion of the vortex lines is fast enough (with respect to the axial motion) to get past the radial center of the condensate where they move axially away from each other due to the reversed velocity field induced by the inhomogeneous density: a rebound takes place, as in Fig. 4(a).

An intermediate regime occurs if, while drifting radially away from the boundary of the condensate towards the center, the minimum distance between the vortices in the central region of the condensate is sufficiently small: in this case, a double reconnection [11] occurs. This happens, for instance, in the sequence in Fig. 4(b), where the two vortex lines touch at a point and exchange their tails both at $\tau=0.208$ and $\tau=0.221$, expressed in units of the precession period $T_{0}$ (see Fig. 7 in Appendix B for a more detailed illustration of the double reconnection dynamics).

In addition to the simulations with chemical potential $\mu=10 \hbar \omega_{\perp}$, we also perform simulations with $\mu=5 \hbar \omega_{\perp}$. The corresponding dynamics are very similar and the sole discriminant parameter between the distinct vortex interaction regimes is indeed the orbit parameter $\chi$. The critical value $\chi_{c}$ switching from rebound to double reconnection dynamics is $0.25<\chi_{c}<0.28$ for $\mu=5$ and $0.22<\chi_{c}<$ 0.25 for $\mu=10$, supporting our argument that the value of $\mu$ does not change the essence of the physics.

\section{INTERPRETATION OF THE RESULTS}

\section{A. Rebounds}

The simulations in Fig. 4(a) show that rebound events are characterized by nonintersecting vortex trajectories, as we observe experimentally in a subset of images, e.g., in
Figs. 3(a) and 3(b). For example, Fig. 4(a) can be related to Fig. 3(b), where the orientations extracted from the residuals start from an orthogonal configuration before partially overlapping (however, the trajectories do not intersect) and then emerge later showing an antiparallel configuration. A simpler, nonrotational, bounce is the one in Fig. 3(a), where vortices are already antiparallel before interacting. Both of the observed rebounds are characterized by an increased visibility when vortices are very close to each other. This is because the residuals are generated by subtracting the unperturbed density distributions and vortices become more visible where their cores lie within a region of higher density. The observed increase of vortex visibility in rebound events is thus consistent with the radial drift of the vortices towards the $x$ axis seen in numerical simulations.

By studying the dynamics of hundreds of different experimental realizations, we make a statistical analysis which reinforces our interpretation. Figure 5(a) shows the distribution of events as a function of the relative axial velocity of two approaching vortices. It is evident that those events, which are identified as rebounds (with approaching, but not touching, trajectories), preferentially happen when the relative velocity is small. As anticipated, the relative angle $\theta_{\text {rel }}$ in the radial plane matters when discerning rebound events from reconnections. Figure 5(b) shows the rebound probability as a function of the vortex relative angle just before their approach. In order to classify the events in the three bins of Fig. 5(b), we use the relation between the shape of the residuals and the orientation of the vortex as extracted from numerical simulations (see Fig. 8 in Appendix C) to postselect all collisions for which we can safely estimate the relative angle to be approximately $0^{\circ}, 45^{\circ}$, and $90^{\circ}$, within an uncertainty of the order of $\sim 30^{\circ}$. Then, in each group we count the fraction of rebounds. The results confirm that rebounds are most likely to occur between antialigned vortices, consistent with the simulations.
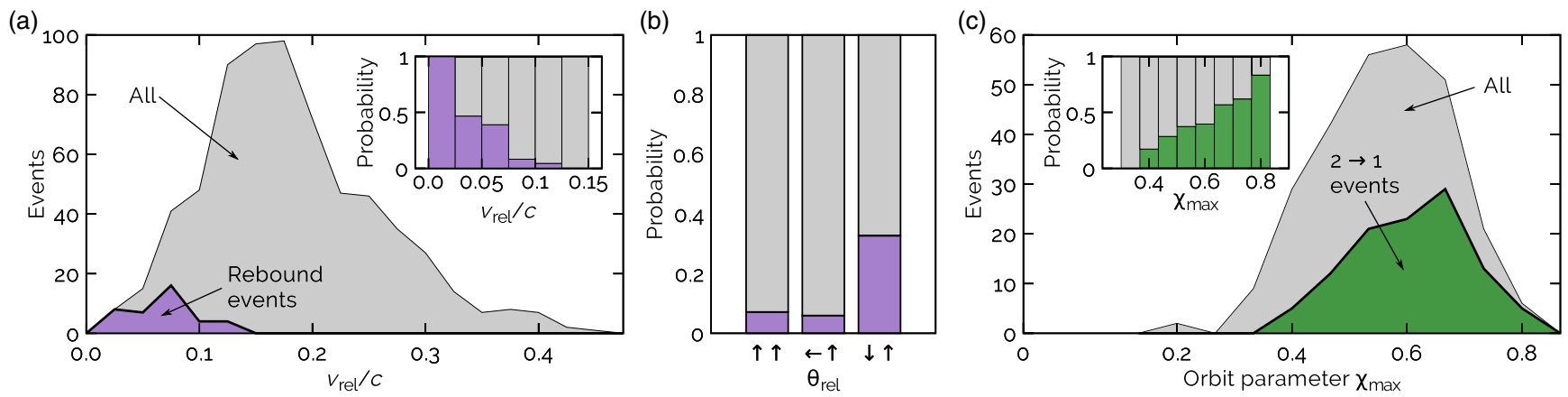

FIG. 5. Statistical analysis of experimental observations. (a) Occurrence of rebound events (purple) as a function of the vortex-vortex relative velocity, within the ensemble of all collision events (gray). The velocity $v_{\text {rel }}$ is normalized to the speed of sound $c$ evaluated at the center of the BEC. The inset shows the relative occurrence for each bin. (b) Fraction of rebound events as a function of the relative angle just before the interaction. (c) Occurrence of events (green) in which one vortex line disappears after the interaction, as a function of the largest orbit parameter of the vortex pair $\chi_{\max }$, i.e., the amplitude of the outer vortex orbit in the BEC. The inset shows the relative occurrence per bin. 


\section{B. Orbiting dynamics}

Two parallel vortices can orbit around the center of the BEC in the same direction with distinct orbit parameters $\chi$, only weakly interacting when they are at the closest distance. When imaged from a radial direction, the two vortices appear to cross periodically; in reality, they pass by each other without visible changes of the residual pattern, with, at most, only slight modifications of orbits and visibility. An example of such orbiting dynamics can be observed in the experimental image Fig. 3(c), and a similar case in the numerical simulations is shown in Fig. 4(e).

\section{Reconnections}

If the initial orientations of the vortices are not parallel and the axial collision dynamics is sufficiently fast, single reconnection processes are favored. As simulations show [Figs. 4(c) and 4(d)], these reconnection processes generate cusps, which, as they relax, form Kelvin waves [67], i.e., helical perturbations of the cores, as, for instance, the ones visible at $\tau=0.192$ in Fig. 4(c). The excitation of Kelvin waves via vortex reconnections was observed in superfluid helium [30], and similar effects have also been found in numerical simulations of Fermi superfluids [68]. In our experiment, such a perturbation of the vortex lines in a reconnection event implies a sudden change of both the orbit and the residual pattern, along with a significant change of visibility of one or both vortices, as illustrated in Figs. 3(d) and 3(e). The nonlinear interaction among Kelvin waves might lead to Kelvin wave cascades [26,27]. However, in the confined geometry of our elongated $\mathrm{BEC}$, the role of Kelvin waves is expected to be reduced compared to a uniform superfluid, due to finite (transverse) size effects. This is consistent with the fact that, if we release the whole condensate from the trap in order to observe the vortex lines by taking absorption images in the axial direction, as done in Refs. [47,57], we typically observe almost rectilinear vortices with only smooth bends (mostly induced by boundary conditions for off-centered vortices), even in the presence of two or more vortices in the condensate.

\section{Ejections}

When a vortex orbits the outer part of the condensate (large $\chi$ ) a fast interaction with another vortex (via either a reconnection or a close orbiting interaction) can cause the expulsion of either vortex towards the surface of the BEC, where the density is too low for observation (the other vortex remaining inside). Examples of such ejection processes are shown in Figs. 3(e) and 3(f) (reconnection induced and orbiting induced, respectively). The numerical counterpart of Fig. 3(e) is illustrated in Fig. 4(d) (in order to emphasize this vortex-visibility effect in the numerical simulations, the thickness and the color of the lines in the plots reported in the right-hand column of Fig. 4 are modulated by the Thomas-Fermi density at which the corresponding vortex core resides; see Appendix B). A statistical analysis of experimental data is given in Fig. 5(c): excluding cases where rebounds occur, we count all events of vortex-vortex interaction as a function of the largest orbit parameter of the vortex pair $\chi_{\max }$. Then, among them, we show in green those in which the visibility of one of the two vortex lines is lost in the interaction. The relative distribution in the inset clearly supports the idea that ejections occur at large $\chi$, i.e., near the boundary of the condensate, in agreement with the result of the numerical simulations. These ejection processes might play a key role in the early postquench dynamics of the BEC, when most of the vorticity produced by the Kibble-Zurek mechanism is progressively lost at the boundaries, eventually leaving only a few vortex lines in the final BEC [69]. It is also worth noticing that a similar dynamics was previously discussed in Ref. [53]. In that case, pairs of dark solitons are created by an optical phase imprinting technique and their subsequent dynamics is observed. GP simulations show that solitons first decay into vortex rings and then into pairs of solitonic vortices, which, in the experimental conditions, are still detected as dark soliton stripes. Hence, a collision between two soliton stripes is actually a collision between two pairs of vortices. Such collisions can be inelastic and can also lead to "sling-shot" events where one of the solitonic vortices is ejected from the condensate. Because of the different mechanism for the creation of vortices, the configurations discussed in Ref. [53] involve typically more than two vortices in each collision, and thus, the dynamics is more complex than in our case, though qualitatively consistent.

\section{CONCLUSIONS}

In conclusion, we develop an innovative experimental technique which, combined with numerical simulations, is capable of determining the real-time position and 3D orientation of vortex lines in an elongated BEC. This combined technique allows us to investigate vortex dynamics in a 3D quantum system with unprecedented resolution: novel types of vortex interaction regimes are unambiguously identified beyond standard reconnections already observed in superfluid helium [29]. While in uniform, unbounded, and nonrotating superfluids reconnections of vortex lines moving towards each other are unavoidable, and their effects have been extensively investigated [31-38], here we show that in a confined and inhomogeneous superfluid, depending on the relative velocity and orientation, two vortex lines can also rebound, perform double reconnections, maintain their orbits with negligible interaction, and undergo ejections. These processes should play even more important roles when the BEC contains more than two vortices, for example, in the case of turbulence [70]. 


\section{ACKNOWLEDGMENTS}

We acknowledge useful discussions with Fabrizio Larcher, Nick Proukakis, George Stagg, and Marek Tylutki. The work of L. G. is supported by Fonds National de la Recherche, Luxembourg, Grant No. 7745104 and by the Italian National Group of Mathematical Physics (GNFMINdAM). C. F. B. acknowledges Grant No. EPSRC EP/ I019413/1. T. B. and F. D. acknowledge the EU QUIC project for financial support. The work was also financially supported by Provincia Autonoma di Trento.

S. S. and L. G. are equally contributed to this work.

\section{APPENDIX A: EXPERIMENTAL PROCEDURE FOR REAL-TIME IMAGING}

Atoms are trapped in a harmonic magnetic trap. The presence of gravity adds a linear potential that shifts the total potential minimum $z_{s} 30 \mu \mathrm{m}$ below the magnetic field minimum. This makes the BEC lie in a region of inhomogeneous magnetic field, mainly varying in the vertical direction $z$. Figure 6 illustrates how the different hyperfine energy levels vary in space because of the second-order Zeeman effect.

The rf field couples the trapped state $|1,-1\rangle$ (green) with the nonmagnetic state $|1,0\rangle$ (red). The resonant frequency is scanned linearly in time from above the BEC to below, at $10 \mathrm{kHz} / \mathrm{ms}$. The position $z_{l}=z_{s}+R_{\perp} \approx 47 \mu \mathrm{m}$ corresponds to the lowest boundary of the condensate.

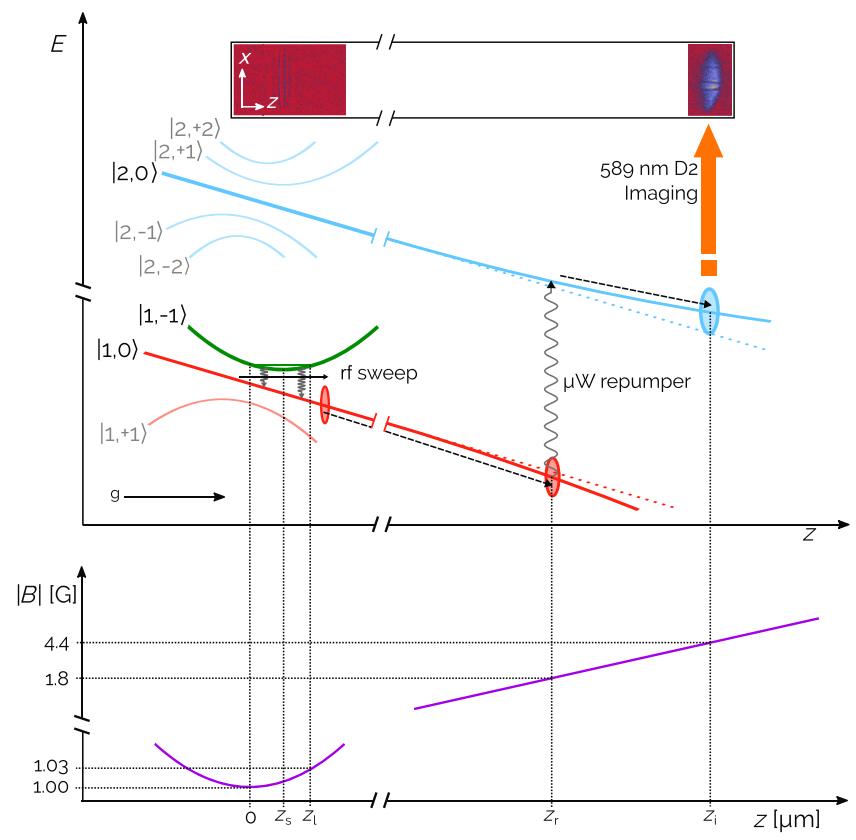

FIG. 6. Schematic picture of the outcoupling technique. In the upper part of the figure, energy levels are reported as a function of the vertical coordinate. In the lower part, the modulus of the trapping magnetic field is reported. The sketched outcoupled atoms in red and cyan are not to scale.
The rf sweep extracts each time a very small fraction of atoms, $\Delta N / N_{0} \approx 1 \%$, and the extraction process is then iterated many times in order to extract the vortex dynamics in real time. The extracted atoms expand and fall freely under the effect of gravity. A microwave field is continuously kept on to couple $|1,0\rangle$ and $|2,0\rangle$ at the position $z_{r} \approx 280 \mu \mathrm{m}$, far enough from the trapped BEC. In this way the extracted falling atoms are transferred to $|2,0\rangle$ as soon as they cross such a surface and become detectable with the D2 probe light, as sketched in Fig. 6. We probe the extracted atoms via standard absorption imaging after $13 \mathrm{~ms}$ of total time of flight at $z_{i} \approx 830 \mu \mathrm{m}$ from the trap center. An example of the experimental image is shown in the inset on the top: only a weak diffraction pattern is visible in the trap region, while the outcoupled atoms become visible through absorption imaging below the repumper surface for $z>z_{r}$, when the sample is promoted to the bright state (cyan).

Experimental images are digitally filtered through a FFT analysis to remove fringes due to the optics elements along the imaging path. The two-dimensional optical density matrix of the sample is integrated along the vertical radial axis $z$ obtaining a one-dimensional axial profile. Such a profile is then fitted with a fourth-order polynomial and residuals are calculated. This procedure is performed on each extraction and then the full temporal sequence is reconstructed in order to follow the vortex trajectories in the trapped condensate. Each sequence is plotted using both a sequential and a diverging color map, to highlight, respectively, the trajectory of vortices and the pattern in the residuals resulting from the self-interference of outcoupled atoms. The axial position of the vortex gives us direct information on the amplitude of the orbit, and hence the orbit parameter $\chi$, as well as on the vortex velocity.

\section{APPENDIX B: NUMERICAL SIMULATIONS OF IN-TRAP DYNAMICS}

Real-time dynamical simulations of a harmonically trapped BEC at $T=0$ are performed by solving the mean-field Gross-Pitaevskii equation,

$$
i \partial_{t} \Psi=\left[-\frac{1}{2} \nabla^{2}+\frac{1}{2}\left(r_{\perp}^{2}+\lambda^{2} x^{2}\right)+\left.\left.\tilde{g}\right|^{\Psi}\right|^{2}\right] \Psi
$$

for the complex macroscopic wave function of the condensate $\Psi=\Psi_{\operatorname{Re}}+i \Psi_{\mathrm{Im}}$. Here, $r_{\perp}=\left(y^{2}+z^{2}\right)^{1 / 2}$ is the radial coordinate and $\lambda=\omega_{x} / \omega_{\perp}$ is the ratio of the axial-toradial trapping frequencies. The mean-field coupling constant is $\tilde{g}=4 \pi N a_{s} / \ell$, where $a_{s}$ is the $s$-wave scattering length and $\ell=\sqrt{\hbar /\left(m \omega_{\perp}\right)}$ is the radial harmonic oscillator length. In the simulations reported in Fig. 4, we use $\lambda=0.2$ and $\tilde{g}=7.4 \times 10^{3}$, which corresponds to $R_{\perp} / \xi=2 \mu /\left(\hbar \omega_{\perp}\right)=20$, where $\mu$ is the chemical potential and $\xi=\hbar / \sqrt{2 m \mu}$ is the healing length. With respect to the experimental setup, in the numerical simulations $\lambda$ is twice as large and the chemical potential $\mu$ is approximately 
3 times smaller. This choice is dictated by the computational resources available, but the key characteristics of the vortex dynamics remain unchanged.

We start the simulation with a Thomas-Fermi parabolic profile for the condensate density $|\Psi|^{2}$. In order to calculate the vortex-free ground state, we evolve the GP equation in imaginary time until the relative decrease of energy $\Delta E / E$ between two consecutive time steps is smaller than the threshold $\epsilon=10^{-5}$. Once this ground state is reached, we numerically imprint the two vortices.

For the numerical simulations illustrated in Figs. 4(a)-4(c), the vortices are initially imprinted in an orthogonal configuration, intersecting the $x$ axis at the points $\left(x_{0}, 0,0\right)$ and $\left(-x_{0}, 0,0\right)$, the first vortex being oriented in the positive $z$ direction, the second vortex in the negative $y$ direction. The corresponding values of the orbit parameter $\chi$ are $0.22,0.25$, 0.375 , for simulations reported in Figs. 4(a)-4(c), respectively. In the simulation illustrated in Fig. 4(d), vortices are also initially orthogonal, but the lower vortex is imprinted in the center of the BEC and oriented in the positive $y$ direction. The orbit parameter $\chi$ of the off-centered vortex is 0.7. Concerning the last simulation, Fig. 4(e), both vortices are oriented in the positive $y$ direction with $\chi=0.33,0.5$.

Vortex imprinting is accomplished by imposing a Padé density profile [71] and a $2 \pi$ phase winding around the vortex axis. We then let the system evolve in imaginary time towards the lowest energy state employing the previously described energy convergence criterion. Once $\Delta E / E<\epsilon$, we start the evolution of the GPE in real time.

Our numerical code employs second-order accurate finite difference schemes to discretize spatial derivatives; the integration in time is performed via a fourth-order Runge-Kutta method. The grid spacings are homogeneous in the three Cartesian directions $(\Delta x=\Delta y=\Delta z=\xi / 3=$ $0.075 \ell$ ) and the time step is $\Delta t=0.00125 \omega_{\perp}^{-1}$. The number of grid points in the $x, y$, and $z$ direction are $\left\{N_{x}, N_{y}, N_{z}\right\}=\{800,224,224\}$, leading to a computational box $\left\{\left[x_{\min }: x_{\max }\right] \times\left[y_{\min }: y_{\max }\right] \times\left[z_{\min }: z_{\max }\right]\right\}=$ $\{[-30: 30] \times[-8.4: 8.4] \times[-8.4: 8.4]\}$, where these values are expressed in units of $\ell$.
Vortex tracking is achieved via an algorithm based on the pseudovorticity unit vector

$$
\hat{\boldsymbol{\omega}}:=\frac{\nabla \Psi_{\mathrm{Re}} \times \nabla \Psi_{\mathrm{Im}}}{\left|\nabla \Psi_{\mathrm{Re}} \times \nabla \Psi_{\mathrm{Im}}\right|},
$$

which is tangent to the vortex line along its length $[38,72]$. To identify the first (starting) point along the axis of each vortex, we use criteria based simultaneously on circulation and density, and then adopt a steepest descent algorithm to achieve subgrid resolution. Successive points on vortex lines are determined with separation distance $\Delta \zeta=\Delta z / 10$.

In the plots reported in the right-hand column of Fig. 4, the initial line colors refer to the colors of the vortices illustrated in the snapshots (red and blue) until a reconnection event occurs. After the latter, the colors employed switch to orange and green and again to red and blue if a second reconnection takes place. The transparency of the lines and the intensity of the colors employed in the plots aim to reproduce the expected experimental vortex visibility. To achieve this aim, we proceed as follows. Since the experimental visibility is obtained by subtracting the background (vortex-free) density profile from the optical integrated densities, the vortex visibility increases for increasing atom number depletion arising from the presence of the vortex itself. As a consequence, both the width and color of the lines plotted in the right-hand column of Fig. 4 are weighted by the value of the Thomas-Fermi density evaluated at the center of vorticity of the corresponding vortex, in order to account, at least qualitatively, for the actual visibility of the vortex in the residual.

Finally, in order to illustrate in more detail the double reconnection dynamics, in Fig. 7 we report radial and axial snapshots of vortex configurations for the double reconnection event already described in Fig. 4(b), but employing a finer temporal resolution. The exchange of vortex strands and the formation of cusps is clearly visible at the reconnection instants $\tau=0.208$ and $\tau=0.221$. After the second reconnection, evidence for the formation of Kelvin waves can be observed (at $\tau=0.224$ ).

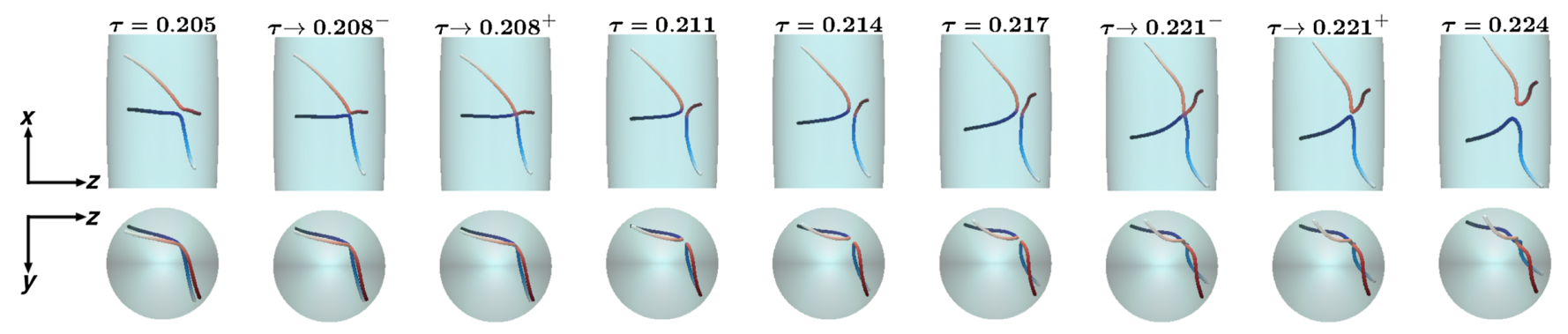

FIG. 7. Temporal sequence of radial (top) and axial (bottom) snapshots from GP simulations showing in detail two interacting vortex lines undergoing a double reconnection. The snapshots refer to the numerical simulation reported in Fig. 4(b), here illustrated employing a finer temporal resolution. 


\section{APPENDIX C: NUMERICAL SIMULATIONS OF EXPANSION}

For the purpose of inferring in situ vortex information from the postexpansion residual densities, we explicitly simulate the outcoupling and expansion dynamically using the GP equation. The outcoupled atoms expand as they fall under gravity relative to the trapped condensate. While the outcoupled atoms may be fairly dilute, they still experience significant interactions with the dense trapped portion, for several milliseconds, until gravity finally separates the components. The corresponding scattering length between outcoupled and trapped atoms is the same as that between trapped atoms, and takes the value 54.54(20) $a_{0}$, where $a_{0}$ is the Bohr radius. Interactions between outcoupled atoms, while less important, are also included, and for these the scattering length is 52.66(40) $a_{0}$ [73].

The partial extraction is performed as a linear-in-energy sweep such that upper atoms are outcoupled before lower atoms. The phase of the trapped component is allowed to evolve during the sweep, which occurs over a few milliseconds, but the in-trap vortex dynamics is much slower and we treat this as fixed. The trapped component's phase, owing to a larger potential energy, evolves more rapidly during the sweep than it does for the released atoms. Consequently, if we consider the example of a horizontal vortex (i.e., oriented in the $y$ direction), by the time the lower atoms are released they have accumulated a greater phase change than the upper ones, which were released earlier, such that these layers interfere constructively on one side of the core and destructively on the other, depending on the sense of the in situ phase circulation. Furthermore, the combined effects of gravity and the intercomponent interactions mean that the speed of the sweep is important. We choose a sweep speed, both experimentally and theoretically, which rapidly compresses the outcoupled cloud in the vertical direction, thus maximizing interference effects. This enhances the $x$-direction asymmetry of the residual, allowing us to determine the orientation and, for horizontal alignment, the sign of the vortex. When extracting vortex information and quantifying this asymmetry, we fit the function

$$
f_{\text {fit }}(x)=\frac{A \cos \left[B\left(x-x_{v}\right)+\delta\right]}{\cosh ^{2}\left[\left(x-x_{v}\right) / C\right]}
$$

to the 1D residual, where $A, B, C, x_{v}$, and $\delta$ are fitting parameters. Here, $x_{v}$ represents the axial position of the vortex while $\delta$ is related to the orientation $\theta$ of the vortex line in the radial plane.

To ensure numerical convergence of the residual to $\sim 1 \%$ when performing a 13-ms expansion, we begin with a grid that initially represents the in situ density, $\left\{x_{\max }, y_{\max }, z_{\max }\right\}=\{51,16,14\} \ell$ and $\left\{N_{x}, N_{y}, N_{z}\right\}=$ $\{300,180,120\}$, and after several interpolations, end with

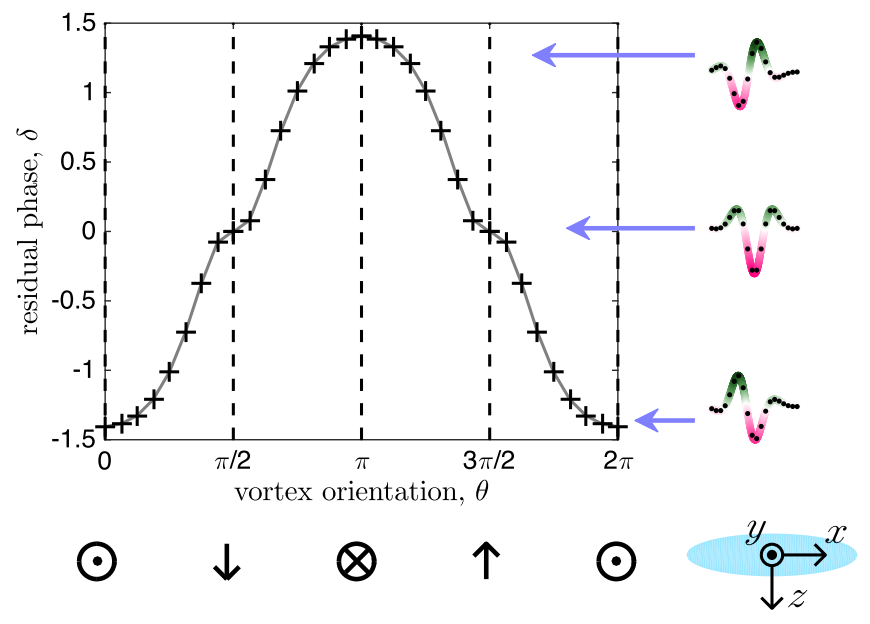

FIG. 8. Residual phase $\delta$ of the extracted portion, after a 13-ms expansion, for various in situ vortex orientations $\theta$, obtained by using the function [Eq. (C1)] to fit the shape of the residuals in GP simulations. These numerical results are for a solitonic vortex stationary state that passes through the condensate center; i.e., even though the vortex is considered for numerous orientations, it is always a straight line that remains in the $x=0$ plane. The $(+)$ symbols are the data points while the solid line guides the eye. For reference, the vertical dashed lines indicate examples of the in situ vortex orientation as indicated by the lower arrows.

a much-enlarged grid, $\left\{x_{\max }, y_{\max }, z_{\max }\right\}=\{75,70,60\} \ell$ and $\left\{N_{x}, N_{y}, N_{z}\right\}=\{180,180,600\}$.

The relation $\delta(\theta)$ is numerically calculated for a straight solitonic vortex and the results are displayed in Fig. 8. Importantly, the fitting function gives a negative value of $\delta$ for a horizontal vortex aligned in the $+y$ direction $(\theta=0)$, whereas the sign of $\delta$ flips for a horizontal vortex of the opposite sense, i.e., $\delta(\theta=\pi)=-\delta(\theta=0)$. For vertically oriented vortices $(\theta=\{\pi / 2,3 \pi / 2\})$, one finds $\delta=0$, and we reiterate here that since every $\delta$ corresponds to two angles, this method cannot, for example, determine the sense of a vertical vortex. We note further that the relationship given by Fig. 8 is expected to be modified for vortices with large orbit parameters.

[1] Shigeo Kida and M. Takaoka, Vortex Reconnection, Annu. Rev. Fluid Mech. 26, 169 (1994).

[2] C. F. Barenghi, R. J. Donnelly, and W. F. Vinen, Quantized Vortex Dynamics and Superfluid Turbulence (Springer Science and Business Media, Berlin, 2001).

[3] K. W. Schwarz, Three-Dimensional Vortex Dynamics in Superfluid ${ }^{4} \mathrm{He}$ : Homogeneous Superfluid Turbulence, Phys. Rev. B 38, 2398 (1988).

[4] E. Priest and T. Forbes, Magnetic Reconnection: MHD Theory and Applications (Cambridge University Press, Cambridge, England, 2007).

[5] H. Che, J. F. Drake, and M. Swisdak, A Current Filamentation Mechanism for Breaking Magnetic Field Lines During Reconnection, Nature (London) 474, 184 (2011). 
[6] J. W. Cirtain et al., Energy Release in the Solar Corona from Spatially Resolved Magnetic Braids, Nature (London) 493, 501 (2013).

[7] I. Chuang, R. Durrer, N. Turok, and B. Yurke, Cosmology in the Laboratory: Defect Dynamics in Liquid Crystals, Science 251, 1336 (1991).

[8] D. W. Sumners, Lifting the Curtain: Using Topology to Probe the Hidden Action of Enzymes, Not. Am. Math. Soc. 42, 528 (1995).

[9] M. Vasquez and D. W. Sumners, Tangles Analysis of Gin Site-Specific Recombination, Math. Proc. Cambridge Philos. Soc. 136, 565 (2004).

[10] M. R. Dennis, R. P. King, B. Jack, K. O’Holleran, and M. J. Padgett, Isolated Optical Vortex Knots, Nat. Phys. 6, 118 (2010).

[11] M. V. Berry and M. R. Dennis, Reconnections of Wave Vortex Lines, Eur. J. Phys. 33, 723 (2012).

[12] L. Onsager, Statistical Hydrodynamics, Nuovo Cimento 6, 279 (1949).

[13] R. P. Feynman, in Progress in Low Temperature Physics, edited by C. J. Gorter (Elsevier, New York, 1955), Vol. 1, p. 17.

[14] W. F. Vinen, The Detection of Single Quanta of Circulation in Liquid Helium II, Proc. R. Soc. A 260, 218 (1961).

[15] R. J. Donnelly, Quantized Vortices in Helium II (Cambridge University Press, Cambridge, England, 1991), p. 346.

[16] C. F. Barenghi, V. L'vov, and P. E. Roche, Experimental, Numerical and Analytical Velocity Spectra in Turbulent Quantum Fluid, Proc. Natl. Acad. Sci. U.S.A. 111, 4683 (2014).

[17] A. W. Baggaley, J. Laurie, and C. F. Barenghi, VortexDensity Fluctuations, Energy Spectra, and Vortical Regions in Superfluid Turbulence, Phys. Rev. Lett. 109, 205304 (2012).

[18] C. Nore, M. Abid, and M. E. Brachet, Kolmogorov Turbulence in Low-Temperature Superflows, Phys. Rev. Lett. 78, 3896 (1997).

[19] J. Maurer and P Tabeling, Local Investigation of Superfluid Turbulence, Europhys. Lett. 43, 29 (1998).

[20] L. Skrbek and K. R. Sreenivasan, Developed Quantum Turbulence and Its Decay, Phys. Fluids 24, 011301 (2012).

[21] N. Navon, A. L. Gaunt, R. P. Smith, and Z. Hadzibabic, Emergence of a Turbulent Cascade in a Quantum Gas, Nature (London) 539, 72 (2016).

[22] V. E. Zakharov, V. S. L'vov, and G. Falkovich, Kolmogorov Spectra of Turbulence I: Wave Turbulence (Springer Science and Business Media, Berlin, 2012).

[23] D. Kleckner, L. H. Kauffman, and W. T. M. Irvine, How Superfluid Vortex Knots Untie, Nat. Phys. 12, 650 (2016).

[24] P. Clark di Leoni, P. D. Mininni, and M. E. Brachet, Helicity, Topology, and Kelvin Waves in Reconnecting Quantum Knots, Phys. Rev. A 94, 043605 (2016).

[25] M. W. Scheeler, D. Kleckner, D. Proment, G. L. Kindlmann, and W. T. M. Irvine, Helicity Conservation by Flow Across Scales in Reconnecting Vortex Links and Knots, Proc. Natl. Acad. Sci. U.S.A. 111, 15350 (2014).

[26] E. Kozik and B. Svistunov, Kelvin-Wave Cascade and Decay of Superfluid Turbulence, Phys. Rev. Lett. 92, 035301 (2004).
[27] E. Kozik and B. Svistunov, Scale-Separation Scheme for Simulating Superfluid Turbulence: Kelvin-Wave Cascade, Phys. Rev. Lett. 94, 025301 (2005).

[28] M. Leadbeater, T. Winiecki, D. C. Samuels, C. F. Barenghi, and C. S. Adams, Sound Emission due to Superfluid Vortex Reconnections, Phys. Rev. Lett. 86, 1410 (2001).

[29] G. P. Bewley, M. S. Paoletti, K. R. Sreenivasan, and D. P. Lathrop, Characterization of Reconnecting Vortices in Superfluid Helium, Proc. Natl. Acad. Sci. U.S.A. 105, 13707 (2008).

[30] E. Fonda, D. P. Meichle, N. T. Ouellette, S. Hormoz, and D. P. Lathrop, Direct Observation of Kelvin Waves Excited by Quantized Vortex Reconnection, Proc. Natl. Acad. Sci. U.S.A. 111, 4707 (2014).

[31] S. Nazarenko and R. West, Analytic Solution for Nonlinear Schrödinger Vortex Reconnection, J. Low Temp. Phys. 132, 1 (2003).

[32] J. Koplik and H. Levine, Vortex Reconnection in Superfluid Helium, Phys. Rev. Lett. 71, 1375 (1993).

[33] A. T. A. M. de Waele and R. G. K. M. Aarts, Route to Vortex Reconnection, Phys. Rev. Lett. 72, 482 (1994).

[34] R. Tebbs, A. J. Youd, and C. F. Barenghi, The Approach to Vortex Reconnection, J. Low Temp. Phys. 162, 314 (2011).

[35] S. Zuccher, M. Caliari, A. W. Baggaley, and C. F. Barenghi, Quantum Vortex Reconnections, Phys. Fluids 24, 125108 (2012).

[36] R. M. Kerr, Vortex Stretching as a Mechanism for Quantum Kinetic Energy Decay, Phys. Rev. Lett. 106, 224501 (2011).

[37] A. Villois, G. Krstulovic, and D. Proment, Universal and Nonuniversal Aspects of Vortex Reconnections in Superfluids, Phys. Rev. Fluids 2, 044701 (2017).

[38] C. Rorai, J. Skipper, R. M. Kerr, and K. R. Sreenivasan, Approach and Separation of Quantum Vortices with Balanced Cores, J. Fluid Mech. 808, 641 (2016).

[39] B. P. Anderson, P. C. Haljan, C. E. Wieman, and E. A. Cornell, Vortex Precession in Bose-Einstein Condensates: Observations with Filled and Empty Cores, Phys. Rev. Lett. 85, 2857 (2000).

[40] C. N. Weiler, T. W. Neely, D. R. Scherer, A. S. Bradley, M. J. Davis, and B. P. Anderson, Spontaneous Vortices in the Formation of Bose-Einstein Condensates, Nature (London) 455, 948 (2008).

[41] D. V. Freilich, D. M. Bianchi, A. M. Kaufman, T. K. Langin, and D. S. Hall, Real-Time Dynamics of Single Vortex Lines and Vortex Dipoles in a Bose-Einstein Condensate, Science 329, 1182 (2010).

[42] T. W. Neely, E. C. Samson, A. S. Bradley, M. J. Davis, and B. P. Anderson, Observation of Vortex Dipoles in an Oblate Bose-Einstein Condensate, Phys. Rev. Lett. 104, 160401 (2010).

[43] P. J. Torres, P. G. Kevrekidis, D. J. Frantzeskakis, R. Carretero-González, P. Schmelcher, and D. S. Hall, Dynamics of Vortex Dipoles in Confined Bose-Einstein Condensates, Phys. Lett. A 375, 3044 (2011).

[44] R. Navarro, R. Carretero-González, P. J. Torres, P. G. Kevrekidis, D. J. Frantzeskakis, M. W. Ray, E. Altuntaş, and D. S. Hall, Dynamics of a Few Corotating Vortices in Bose-Einstein Condensates, Phys. Rev. Lett. 110, 225301 (2013). 
[45] J. Brand and W. P. Reinhardt, Solitonic Vortices and the Fundamental Modes of the Snake Instability: Possibility of Observation in the Gaseous Bose-Einstein Condensate, Phys. Rev. A 65, 043612 (2002).

[46] S. Komineas and N. Papanicolaou, Solitons, Solitonic Vortices, and Vortex Rings in a Confined Bose-Einstein Condensate, Phys. Rev. A 68, 043617 (2003).

[47] S. Donadello, S. Serafini, M. Tylutki, L. P. Pitaevskii, F. Dalfovo, G. Lamporesi, and G. Ferrari, Observation of Solitonic Vortices in Bose-Einstein Condensates, Phys. Rev. Lett. 113, 065302 (2014).

[48] M. Tylutki, S. Donadello, S. Serafini, L. P. Pitaevskii, F. Dalfovo, G. Lamporesi, and G. Ferrari, Solitonic Vortices in Bose-Einstein Condensates, Eur. Phys. J. Spec. Top. 224, 577 (2015).

[49] T. W. B. Kibble, Topology of Cosmic Domains and Strings, J. Phys. A 9, 1387 (1976).

[50] W. H. Zurek, Cosmological Experiments in Condensed Matter Systems, Phys. Rep. 276, 177 (1996).

[51] G. Lamporesi, S. Donadello, S. Serafini, F. Dalfovo, and G. Ferrari, Spontaneous Creation of Kibble-Zurek Solitons in a Bose-Einstein Condensate, Nat. Phys. 9, 656 (2013).

[52] S. Donadello, S. Serafini, T. Bienaimé, F. Dalfovo, G. Lamporesi, and G. Ferrari, Creation and Counting of Defects in a Temperature-Quenched Bose-Einstein Condensate, Phys. Rev. A 94, 023628 (2016).

[53] C. Becker, K. Sengstock, P. Schmelcher, P. G. Kevrekidis, and R. Carretero-González, Inelastic Collisions of Solitary Waves in Anisotropic Bose-Einstein Condensates: SlingShot Events and Expanding Collision Bubbles, New J. Phys. 15, 113028 (2013).

[54] M. J. H. Ku, W. Ji, B. Mukherjee, E. Guardado-Sanchez, L. W. Cheuk, T. Yefsah, and M. W. Zwierlein, Motion of a Solitonic Vortex in the BEC-BCS Crossover, Phys. Rev. Lett. 113, 065301 (2014).

[55] M. J. H. Ku, B. Mukherjee, T. Yefsah, and M. W. Zwierlein, Cascade of Solitonic Excitations in a Superfluid Fermi Gas: From Planar Solitons to Vortex Rings and Lines, Phys. Rev. Lett. 116, 045304 (2016).

[56] A. Ramanathan, S. R. Muniz, K. C. Wright, R. P. Anderson, W. D. Phillips, K. Helmerson, and G. K. Campbell, PartialTransfer Absorption Imaging: A Versatile Technique for Optimal Imaging of Ultracold Gases, Rev. Sci. Instrum. 83, 083119 (2012).

[57] S. Serafini, M. Barbiero, M. Debortoli, S. Donadello, F. Larcher, F. Dalfovo, G. Lamporesi, and G. Ferrari, Dynamics and Interaction of Vortex Lines in an Elongated Bose-Einstein Condensate, Phys. Rev. Lett. 115, 170402 (2015).
[58] G. P. Bewley, D. P. Lathrop, and K. R. Sreenivasan, Visualization of Quantized Vortices, Nature (London) 441, 588 (2006).

[59] L. Pitaevskii and S. Stringari, Bose-Einstein Condensation and Superfluidity (Oxford University Press, New York, 2016).

[60] F. Dalfovo, S. Giorgini, L. P. Pitaevskii, and S. Stringari, Theory of Bose-Einstein Condensation in Trapped Gases, Rev. Mod. Phys. 71, 463 (1999).

[61] A. J. Allen, S. Zuccher, M. Caliari, N. P. Proukakis, N. G. Parker, and C. F. Barenghi, Vortex Reconnections in Atomic Condensates at Finite Temperature, Phys. Rev. A 90, 013601 (2014).

[62] A. A. Svidzinsky and A. L. Fetter, Stability of a Vortex in a Trapped Bose-Einstein Condensate, Phys. Rev. Lett. 84, 5919 (2000).

[63] E. Lundh and P. Ao, Hydrodynamic Approach to Vortex Lifetimes in Trapped Bose Condensates, Phys. Rev. A 61, 063612 (2000).

[64] D. E. Sheehy and L. Radzihovsky, Vortices in Spatially Inhomogeneous Superfluids, Phys. Rev. A 70, 063620 (2004).

[65] A. L. Fetter, Rotating Trapped Bose-Einstein Condensates, Rev. Mod. Phys. 81, 647 (2009).

[66] S. Middelkamp, P. J. Torres, P. G. Kevrekidis, D. J. Frantzeskakis, R. Carretero-González, P. Schmelcher, D. V. Freilich, and D. S. Hall, Guiding-Center Dynamics of Vortex Dipoles in Bose-Einstein Condensates, Phys. Rev. A 84, 011605(R) (2011).

[67] D. Kivotides, J. C. Vassilicos, D. C. Samuels, and C. F. Barenghi, Kelvin Waves Cascade in Superfluid Turbulence, Phys. Rev. Lett. 86, 3080 (2001).

[68] G. Wlazłowski, A. Bulgac, Michael McNeil Forbes, and K. J. Roche, Life Cycle of Superfluid Vortices and Quantum Turbulence in the Unitary Fermi Gas, Phys. Rev. A 91, 031602 (2015).

[69] I-Kang Liu et al. (to be published).

[70] M. C. Tsatsos, P. E. S. Tavares, A. Cidrim, A. R. Fritsch, M. A. Caracanhas, F. E. A. dos Santos, C. F. Barenghi, and V.S. Bagnato, Quantum Turbulence in Trapped Atomic Bose-Einstein Condensates, Phys. Rep. 622, 1 (2016).

[71] N. G. Berloff, Padé Approximations of Solitary Wave Solutions of the Gross-Pitaevskii Equation, J. Phys. A 37, 1617 (2004).

[72] A. Villois, G. Krstulovic, D. Proment, and H. Salman, A Vortex Filament Tracking Method for the Gross-Pitaevskii Model of a Superfluid, J. Phys. A 49, 415502 (2016).

[73] S. Knoop, T. Schuster, R. Scelle, A. Trautmann, J. Appmeier, M. K. Oberthaler, E. Tiesinga, and E. Tiemann, Feshbach Spectroscopy and Analysis of the Interaction Potentials of Ultracold Sodium, Phys. Rev. A 83, 042704 (2011). 